\title{
LV-EV-4
}

\section{Laparoscopic living donor hepatectomy using the left liver - pushing the limits further}

\author{
Choon Hyuck David KWON*
}

Cleveland Clnic, Cleveland, OH, USA

Lecture: With the accumulation of experience in both living donor liver transplantation (LDLT) and laparoscopic liver surgery, efforts have been made to minimize the surgical trauma to the donor and many institutions are now using minimally invasive approach for donor surgery. Nevertheless, the overwhelming majority of the grafts used for adult LDLT are right lobe grafts. Right lobe, which usually consist of $2 / 3$ of the liver, provides a larger size grafts, facilitating the recovery of the recipient but this comes at the price of the increase risk of the donor. It is known that utilization of right lobe has a higher complication and mortality rate compared to the left, but the utilization of the left lobe has not been practiced widely. In this talk, I'll provide the early results of our program using laparoscopic approach for left lobe and describe the detailed techniques through video presentation. 